\title{
Ultrasound Localization of Intercostal Perforating Vessels Reduces Skin Necrosis Complications from Nipple-Sparing Mastectomy
}

\author{
Robert Maganini, MD, FACs ${ }^{1 *}$, Anthony Maganini ${ }^{2}$, Richard Maganini $^{3}$ \\ ${ }^{1}$ Amita Health System, Bartlett, IL, USA \\ ${ }^{2}$ Undergraduate Studies, University of Miami, Coral Gables, FL, USA \\ ${ }^{3}$ Undergraduate Studies, Northwestern University, Evanston, IL, USA
}

${ }^{\star}$ Corresponding to: Robert Maganini, MD, FACS, Amita Health System, Bartlett, IL, USA; Phone: 224-273-5120; Fax: 224-273-5349; Email: robert.maganini@amitahealth.org Received: June 06, 2019; Accepted: June 20, 2019; Published: June 28, 2019;

\section{Synopsis}

Skin necrosis of the nipple-areolar complex (NAC), as a result of ischemia is a well-documented complication of nipple-sparing mastectomy. Ultrasound localization of the intercostal perforating vessels, which are essential to NAC viability, can reduce necrosis complications.

\section{Abstract \\ Background}

Complications of nipple-sparing mastectomy (NSM) are well documented, particularly skin necrosis of the nipple-areolar complex (NAC), as a result of ischemia. The intercostal perforating vessels (IPV) provide a significant proportion of the blood supply to the NAC. We propose that ultrasound (US) localization of the IPV will reduce the incidence and severity of NAC necrosis following NSM.

\section{Methods}

168 mastectomies were performed in 101 patients between Feb. 2015 and Feb. 2017. In Feb. 2016, prospective data was collected in 35 NSM with US localization of the IPV. Rate and severity of skin or NAC necrosis was objectively measured using the skin ischemic necrosis (SKIN) score. Retrospective data and SKIN score were obtained on 52 consecutive historical control NSM from the prior 12 month period.

\section{Results}

20 women underwent 35 NSM with US localization. Preservation of the localized IPV was $100 \%$ with significant variability observed in the intercostal space location. Comparison of SKIN scores between the study group and historical controls demonstrated a trend towards fewer and less severe complications with utilization of US localization. Grouping clinically significant complications together for statistical comparison with no or minimal complications did not show statistical significance $(p=0.77)$.

\section{Conclusions}

Our prospective study demonstrates that US localization of the IPV can reduce NAC and skin necrosis complications in NSM. Although grouping of the data into clinically meaningful categories did not show evidence of statistical significance, reduced rates of necrosis, as measured by SKIN score, were observed.

\section{Introduction}

Complications of nipple-sparing Mastectomy (NSM) are well documented, particularly skin necrosis of the nipple-areolar complex (NAC), as a result of ischemia. NAC necrosis rates are reported in the literature from $20-43 \% 1$ [1-3]. Carlson et al. reported a $28.2 \%$ rate of partial nipple necrosis in 71 consecutive NSM [1]. Cho et al. performed an analysis of 85 women undergoing NSM [2]. Thirty-six total cases of NAC necrosis occurred at a rate of $43.4 \%$ among the patients. A nipple necrosis rate of $20 \%$ was reported by Gould et al. in a series of 113 NSM [3]. Risk factors for NAC necrosis include: age, smoking, prior radiation therapy (RT), body mass index (BMI), incision location, and cancer versus prophylactic procedure [1-4].

The intercostal perforating vessels (IPV) have been demonstrated to provide a significant proportion of the blood supply to the NAC [4-7]. O’Dey et al. performed a microdissection study to better understand vascular reliability of NAC pedicles on six different 
arterial sources: the internal mammary artery, the highest thoracic artery, the anterior and posterior intercostal artery branches, the thoracoacromial artery, the superficial thoracic artery, and the lateral thoracic artery [8]. While acknowledging the considerable variability among individuals in arterial development and vascular reliability of the NAC, this study produced findings consistent with othersnamely, in most cases the lateral thoracic and internal mammary arteries are the most important suppliers of blood to the NAC. The complexity and variability of NAC vascularity are well-demonstrated in the comprehensive review of anatomical studies by van Deventer and Graewe [9]. They emphasize in their review that an awareness of the vascular anatomy and its variability will be helpful to reduce the risk of NAC necrosis.

To our knowledge, no data has been published on US localization of the vasculature to the NAC to reduce skin necrosis in NSM. A recent report suggested that identification of blood supply to the NAC on preoperative MRI could limit necrosis rates in NSM [10].

Our objective is to reduce the significant rates of NAC necrosis observed in NSM, even in lower risk individuals. We propose that US localization of the IPV will reduce the incidence and severity of NAC necrosis following NSM.

\section{Materials and Methods}

Approval for our study was obtained from our local IRB. 168 mastectomies were performed by the author in 101 patients between Feb. 2015 and Feb. 2017. Of these, 90 NSM (54\%) were performed in 51 patients. Starting in Feb. 2016, prospective data collection was performed in all women scheduled for NSM and providing consent for the study. US was performed at the beginning of the procedure, with a Sonosite ${ }^{\text {Th }}$ US, 5-12 $\mathrm{mHz}$ transducer, using the Doppler mode. The IPV were identified by scanning each intercostal space near the sternal border. If an IPV was clearly identified (Figure 1), the skin was marked at that location as an aid to the surgeon during dissection. All incisions were performed in the lateral infra-mammary fold (IMF). Data monitored included: age, smoking history, BMI, prior RT, reconstruction technique, success of localization, location of IPV, and success of preservation of IPV during the procedure. Rate and severity of necrosis of the skin or NAC was objectively measured using the skin ischemic necrosis (SKIN) score [11]. Retrospective data was collected from our dedicated breast cancer database and electronic medical records on 52 consecutive NSM in 29 patients from the prior 12 month period to provide a historical control group. Statistical analysis was performed comparing none or superficial necrosis (SKIN A1 and B2) with major necrosis (SKIN B3, C2, C3, D3) in the historical controls versus the study group using Fisher's Exact Test.

\section{Results}

38 NSM were performed in 22 patients during the study period. Two women (3 NSM) (8\%) declined participation in the study, thus, the study group consisted of 20 women undergoing 35 NSM who had US for localization of the IPV. The indication for surgery overall was malignancy in 18 (51\%) and prophylaxis in 17 (49\%). The control and study groups were well-matched for known risk factors including: age, prior RT, history of smoking (current or $>10$ pk./yrs.),
BMI, reconstruction technique, and indication (Table 1). Localization was successful in 33 (96\%), the two failed localizations occurred in the same woman. Preservation of the localized IPV was $100 \%$. We identified significant variability in the location of the primary IPV: nearly equal frequency in the 2 nd intercostal space (ICS) (37\%) and 3rd ICS (34\%); multiple ICS locations in $14.3 \%$, and less commonly, in the 1st ICS (8.6\%) (Table 2). These findings are consistent with previously published anatomical studies [5-9]. Complication rates and severity, as measured by SKIN score, are shown in Table 3. Rates of necrosis observed in the control group were consistent with the published literature [1-3]. No NAC or skin necrosis was observed in $63 \%$ of the study group compared with $42 \%$ of the controls. A SKIN score of B2, representing minimal, uncomplicated superficial necrosis, was observed in $23 \%$ of women having US localization versus $38 \%$ in the control group. Rates of more significant necrosis (SKIN scores B3, C2, C3, \& D3) were found in $14 \%$ of the study cases compared with $20 \%$ of the controls. The data demonstrates a trend towards fewer and less severe complications in NSM cases utilizing US localization of the IPV. We elected to group more clinically significant complications (SKIN B3, C2, C3, \& D3) together for statistical comparison with no or minimal complications (SKIN A1 \& B2). The results of this analysis are presented in Table 4 . No statistically significant difference was observed in this analysis. $(p=0.77)$

Table 1. Risk Factor Matching

\begin{tabular}{|r|c|c|}
\hline & Study & Control \\
\hline Age (average) & 45.1 & 46.0 \\
\hline (standard deviation) & 8.0 & 8.7 \\
\hline Age $>65$ & 2 & 1 \\
\hline BMI (average) & 24.4 & 24.8 \\
\hline (standard deviation) & 4.1 & 5.1 \\
\hline Prior RT & $6 \%$ & $6 \%$ \\
\hline Tissue Expander & $83 \%$ & $83 \%$ \\
\hline Direct Implant & $14 \%$ & $17 \%$ \\
\hline Autologous & $3 \%$ & $0 \%$ \\
\hline Indication & & \\
\hline Malignancy & $51 \%$ & $54 \%$ \\
\hline Prophylaxis & $49 \%$ & $46 \%$ \\
\hline (Current or $>10$ pack/years) & $14 \%$ & $13 \%$ \\
\hline
\end{tabular}

\section{Discussion}

Previous evidence suggests that skin and NAC necrosis rates are significant in the range of $20-40 \%$ [1-3]. Our personal experience with these complications is that many occurrences are superficial and resolve with minimal intervention, however a significant proportion are significant and can have devastating effects. Risk factors are well established for NAC necrosis in NSM [1-4]. We have selected NSM candidates carefully for these risk factors in our practice as 
evidenced by the younger age, lower incidence of smoking history or prior RT, relatively lower BMI, and use of infra-mammary incisions. Our objective was to monitor our NAC necrosis complications and continually adopt or develop methodologies to reduce these complications.

Table 2. Ultrasound Localization of Intercostal Perforator Vessel

\begin{tabular}{|r|c|c|}
\hline \multicolumn{3}{|c|}{ Localization Success } \\
\hline Yes & 33 & $96 \%$ \\
\hline No & 2 & $4 \%$ \\
\hline 3 & 13 & $37.1 \%$ \\
\hline 2 & 12 & $34 \%$ \\
\hline 1 & 3 & $8.6 \%$ \\
\hline Primary ICS Location \\
\hline Multiple & 5 & $14.3 \%$ \\
\hline NA & 2 & $5.7 \%$ \\
\hline
\end{tabular}

Table 3. Skin ischemic necrosis (SKIN) score

\begin{tabular}{|c|c|c|c|c|c|}
\hline \multicolumn{2}{|c|}{$\begin{array}{c}\text { SKIN Score } \\
\text { n }\end{array}$} & \multicolumn{2}{c|}{ Study } & \multicolumn{2}{c|}{ Control } \\
\cline { 2 - 6 } & $\%$ & $\mathbf{n}$ & $\%$ & \\
\hline \multirow{2}{*}{$\begin{array}{c}\text { None or } \\
\text { Minor }\end{array}$} & None (A1) & 22 & $63 \%$ & 22 & $42 \%$ \\
\cline { 2 - 6 } & B2 & 8 & $23 \%$ & 20 & $38 \%$ \\
\hline \multirow{2}{*}{ Major } & B3 & 4 & $11 \%$ & 6 & $12 \%$ \\
\cline { 2 - 6 } & C2 & 1 & $3 \%$ & 3 & $2 \%$ \\
\cline { 2 - 6 } & C3 & 0 & $0 \%$ & 0 & $0 \%$ \\
\cline { 2 - 6 } & D3 & 0 & $0 \%$ & 1 & $6 \%$ \\
\hline
\end{tabular}

Table 4. Statistical analysis of none or minor necrosis with major necrosis

\begin{tabular}{|c|c|c|c|c|c|}
\hline \multicolumn{3}{|c|}{$\begin{array}{l}\text { None or superficial necrosis (A1 or B2) } \\
\text { Major necrosis }(\mathrm{B} 3, \mathrm{C} 2, \mathrm{C} 3, \mathrm{D} 3)\end{array}$} & \multicolumn{2}{|c|}{$\begin{array}{l}\text { SKIN Score } \\
\text { Category }\end{array}$} & \multirow{2}{*}{$\begin{array}{c}\text { Total } \\
\\
52\end{array}$} \\
\hline \multirow{4}{*}{$\begin{array}{c}\text { Use of } \\
\text { Ultrasound } \\
\text { in the Nipple } \\
\text { Sparing } \\
\text { Mastectomy? }\end{array}$} & No & n & $42^{\mathrm{a}}$ & $10^{\mathrm{a}}$ & \\
\hline & $\begin{array}{l}\text { (Control } \\
\text { Group) }\end{array}$ & $\%$ & $80.8 \%$ & $19.2 \%$ & $100.0 \%$ \\
\hline & Yes & $\mathbf{n}$ & $30^{\mathrm{a}}$ & $5^{\mathrm{a}}$ & 35 \\
\hline & $\begin{array}{l}\text { (Study } \\
\text { Cohort) }\end{array}$ & $\%$ & $85.7 \%$ & $14.3 \%$ & $100.0 \%$ \\
\hline \multirow{2}{*}{\multicolumn{2}{|c|}{$\begin{array}{c}\text { Total } \\
\%\end{array}$}} & $\mathrm{n}$ & 72 & 15 & 87 \\
\hline & & $82.8 \%$ & $17.2 \%$ & $100.0 \%$ & \\
\hline
\end{tabular}

a denotes subset of SKIN Score Category categories whose column proportions do not differ significantly from each other at the 0.05 level. Fisher's Exact Test: $p=0.77$

The importance of the IPV to reducing NAC complications is historically founded in the plastic surgery literature as relates to developing vascular pedicles for aesthetic surgery. The advent of contemporary NSM reintroduced the importance of preservation of the IPV to reduce NAC complications. Recognizing the importance of the IPV and well-documented variability, we developed the US localization procedure to aid in the identification and preservation of these vessels to reduce NAC complications in NSM.

Our study has several limitations. Although we have a prospective design, use of historical controls introduces potential inaccuracies related to retrospective data collection. We did not control for several different plastic surgeons and the potential for variability in their technique including fill volume of tissue expanders. We did not examine cost effectiveness of our technique. However, we believe that reducing NAC necrosis complications is inherently cost effective. Lastly, in our statistical analysis of two grouped categories, no or minor complications versus major complications, we did not achieve statistical significance.

We did demonstrate a reduction in the number of women experiencing necrosis and more severe types of necrosis as measured by SKIN score. Localization was highly successful and performed with equipment already available in the operating room. Many surgeons who regularly perform breast surgery are adept at US and would be capable of readily employing this technique.

\section{Conclusions}

Our prospective study provides preliminary evidence that US localization of the IPV can reduce NAC and skin necrosis complications in NSM. Localization of the IPV was highly successful with US suggesting that surgeons facile with breast US can readily employ this technique to reduce skin necrosis and related complications. The observed variable location of the primary IPV, consistent with previously published literature, contributes to the value of localization. Although grouping of the data into clinically meaningful categories did not show evidence of statistical significance, reduced rates of necrosis, as measured by SKIN score, were observed in women undergoing NSM with US localization of the IPV. The findings are consistent with our hypothesis that localization of the IPV leads to preservation of the primary vasculature of the NAC and reduced necrosis. Future efforts will be directed at performing US localization pre-operatively to improve efficiency, incorporation of indocyanine green fluorescence angiography to further reduce necrosis rates of NSM, and examining the effect of pre-pectoral implant placement.

\section{Acknowledgment}

We would like to acknowledge Todd Foster, Isaac Janson, and Jessica Wolfe, St. Vincent Hospital, Indianapolis, IN for their input and kind assistance with the statistical analysis.

\section{References}

1. Carlson GW, Chu CK, Moyer HR, Duggal C, Losken A. (2014) Predictors of nipple ischemia after nipple sparing mastectomy. Breast J. 20(1): 69-73. [Crossref]

2. Cho JW, Yoon ES, You HJ, Kim HS, Lee BI, Park SH. (2015) Nipple-Areola Complex Necrosis after Nipple-Sparing Mastectomy with Immediate Autologous Breast Reconstruction. Arch Plast Surg. 42(5): 601-7. [Crossref]

3. Gould DJ, Hunt KK, Liu J, Kuerer HM, Crosby MA, Babiera G, Kronowitz SJ (2013) Impact of surgical techniques, biomaterials, and patient variables on rate of nipple necrosis after nipple-sparing mastectomy. Plast Reconstr Surg. 132(3): 330e-8e. [Crossref] 
Robert Maganini (2019) Ultrasound Localization of Intercostal Perforating Vessels Reduces Skin Necrosis Complications from Nipple-Sparing Mastectomy

4. Frey JD, Alperovich M, Levine JP, Choi M, Karp NS. (2017) Does Smoking History Confer a Higher Risk for Reconstructive Complications in Nipple-Sparing Mastectomy? Breast J. [Crossref]

5. Stone K, Wheeler A. (2015) A Review of Anatomy, Physiology, and Benign Pathology of the Nipple. Ann Surg Oncol 22: 3236-3240. [Crossref]

6. Skandalakis J. Breast. In: Skandalakis J, editor. (2004) Skandalakis' surgical anatomy: the embryologic and anatomic basis of modern surgery. Athens: PMP; pp. 106-107.

7. Palmer JH, Taylor GI. (1986) The vascular territories of the anterior chest wall. $\mathrm{Br}$ J Plast Surg. 39: 287-99. [Crossref]

8. O'Dey D, Prescher A, Pallua N. (2007) Vascular reliability of nipple-areola complex-bearing pedicles: an anatomical microdissection study. Plast Reconstr
Surg. 119(4): 1167-77. [Crossref]

9. van Deventer PV, Graewe FR. (2016) The Blood Supply of the Breast Revisited. Plast Reconstr Surg. 137(5): 1388-97. [Crossref]

10. Bahl M, Pien I, Buretta K, Hwang S, Greenup, R, Ghate S, Hollenbeck S. (2016) Can Vascular Patterns on Preoperative Magnetic Resonance Imaging Help Predict Skin Necrosis after Nipple-Sparing Mastectomy? J Am Coll Surg 223(2): 279-85. [Crossref]

11. Lemaine V, Hoskin T, Farley D, Grant C, Boughey J, Torstenson T, Jacobson S, et al. (2015) Introducing the SKIN Score: A Validated Scoring System to Assess Severity of Mastectomy Skin Flap Necrosis. Ann Surg Oncol 22: 2925-2932. [Crossref]

Citation:

Robert Maganini, Anthony Maganini, Richard Maganini (2019) Ultrasound Localization of Intercostal Perforating Vessels Reduces Skin Necrosis Complications from Nipple-Sparing Mastectomy. J Clin Res Med Volume 2(3): 1-4 